\title{
Altered pancreas remodeling following glucose intolerance in pregnancy in mice
}

\author{
Sandra K Szlapinski1,2, Anthony A Botros ${ }^{1,2}$, Sarah Donegan1,2, Renee T King², Gabrielle Retta², Brenda J Strutt1,2 and \\ David J Hill1,2
}

1Department of Physiology and Pharmacology, Western University, London, Ontario, Canada

2Lawson Health Research Institute, St Joseph's Health Care, London, Ontario, Canada

Correspondence should be addressed to S K Szlapinski: sszlapin@uwo.ca

\begin{abstract}
Gestational diabetes mellitus increases the risk of dysglycemia postpartum, in part, due to pancreatic $\beta$-cell dysfunction. However, no histological evidence exists comparing endocrine pancreas after healthy and glucose-intolerant pregnancies. This study sought to address this knowledge gap, in addition to exploring the contribution of an inflammatory environment to changes in endocrine pancreas after parturition. We used a previously established mouse model of gestational glucose intolerance induced by dietary low protein insult from conception until weaning. Pancreas and adipose samples were collected at 7, 30 and 90 days postpartum for histomorphometric and cytokine analyses, respectively. Glucose tolerance tests were performed prior to euthanasia and blood was collected via cardiac puncture. Pregnant female mice born to dams fed a low protein diet previously shown to develop glucose intolerance at late gestation relative to controls continued to be glucose intolerant until 1 month postpartum. However, glucose tolerance normalized by 3 months postpartum. Glucose intolerance at 7 days postpartum was associated with lower beta- and alpha-cell fractional areas and higher adipose levels of pro-inflammatory cytokine, interleukin-6. By 3 months postpartum, a compensatory increase in the number of small islets and a higher insulin to glucagon ratio likely enabled euglycemia to be attained in the previously glucose-intolerant mice. The results show that impairments in endocrine pancreas compensation in hyperglycemic pregnancy persist after parturition and contribute to prolonged glucose intolerance. These impairments may increase the susceptibility to development of future type 2 diabetes.
\end{abstract}

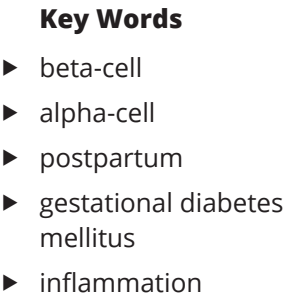

Journal of Endocrinology (2020) 245, 315-326

\section{Introduction}

Pregnancy presents as a unique situation of endocrine pancreas $\beta$-cell adaptation that reverses after parturition. Late pregnancy is characterized by a state of peripheral maternal insulin resistance mediated by placental hormones (Newbern \& Freemark 2011) which is essential for maintaining trans-placental transport of glucose to the fetus for optimal development. To compensate for insulin resistance, the maternal pancreas responds by increasing endocrine pancreatic $\beta$-cell mass (BCM) to help maintain euglycemia. An adaptive expansion of BCM has been documented in both rodents and humans and is maximal at late gestation (around gestational day (GD) 18 in rodents) (Van Assche et al. 1978, Parsons et al. 1992, Sorenson \& Brelje 1997, Butler et al. 2010, 
Beamish et al. 2017, Szlapinski et al. 2019). The adaptive mechanisms of BCM expansion during rodent pregnancy have been studied extensively and occur, in part, due to increased $\beta$-cell hypertrophy and proliferation, and the expansion of a $\beta$-cell precursor pool, all of which peak at mid-gestation (Parsons et al. 1992, Rieck \& Kaestner 2010, Beamish et al. 2017, Szlapinski et al. 2019). These changes are mediated by increased levels of hormones including placental lactogen, prolactin, and estrogen (Rieck \& Kaestner 2010, Newbern \& Freemark 2011). After parturition, progesterone-mediated $\beta$-cell apoptosis increases while proliferation decreases concomitant with an absence of placental lactogen, returning BCM to prepregnancy levels (Scaglia et al. 1995, Rieck \& Kaestner 2010). If BCM expansion is sub-optimal, gestational diabetes mellitus (GDM) can arise.

GDM is described as diabetes that first appears during pregnancy and occurs due to insufficient $\beta$-cell adaptation to compensate for insulin resistance in pregnancy. The incidence of GDM worldwide is around $7-10 \%$ of all pregnancies (Ferrara 2007, Nguyen et al. 2018), although the incidence will vary depending on the population characteristics and diagnostic criteria. As more women enter pregnancy obese or at an older age, the incidence of GDM is projected to continue to rise (Petra et al. 2019). An increased incidence of GDM is associated with morbidity due to adverse fetal outcomes (Garcia-Vargas et al. 2012, Kim et al. 2012, Mitanchez et al. 2015) and adverse longterm maternal outcomes including an increased risk of type-2 diabetes mellitus (T2DM) (Heida et al. 2015) between 3\% to 90\% (Melchior et al. 2017). Clinical studies have shown that glucose intolerance after parturition involves $\beta$-cell dysfunction (Buchanan \& Xiang 2005, Retnakaran et al. 2008, Xiang et al. 2010, Molęda et al. 2013) which can occur, in part, due to inflammation and glucotoxicity-induced oxidative stress in $\beta$-cells (Tang et al. 2007).

Pregnancy can be characterized as a low-grade inflammatory state where the placenta is a major source of cytokines that can alter $\beta$-cell function (Wedekind \& Belkacemi 2016). For example, studies have postulated that $94 \%$ of the increased serum levels of tumor necrosis factor (TNF- $\alpha$ ) in women during pregnancy results from placental production (Pantham et al. 2015). The first and early second trimester is a pro-inflammatory environment characterized by helper T-cell-1 (Th1) cytokines, such as TNF- $\alpha$, interleukin-1 $\beta$, (IL-1 $\beta$ ), and interferon (IFN)- $\gamma$, resulting from implantation and placentation processes (Wedekind \& Belkacemi 2016). The second trimester is characterized as an anti-inflammatory environment with Th2 cytokines that permit for fetal growth and development (Mor et al. 2011). Lastly, the third trimester is characterized by the recurrence of a pro-inflammatory environment in preparation for parturition, as inflammation promotes uterine contractions (Gibbs et al. 1992). Thus, increased cytokine production occurs in healthy pregnancies. However, increased activity of the immune-checkpoint molecule programmed deathligand 1 (PD-L1) and its receptor, PD-1, play a role in the maintenance of immunological balance between mother and fetus (Meggyes et al. 2019) and likely protect maternal $\beta$-cells from cytotoxic damage. In healthy pregnancies, PD-L1 is expressed by syncytiotrophoblast cells of the placenta in response to pro-inflammatory stimuli and is increasingly released into the maternal bloodstream as gestation progresses (Veras et al. 2017). The interaction between PD-L1 and PD-1 reduces clonal expansion of pathogenic lymphocytes and their associated cytokine release (Wang et al. 2008). Therefore, with decreased lymphocyte expansion, cytokine production is decreased and $\beta$-cells are protected against cytokine-induced damage. In the context of GDM and persistent glucose intolerance at postpartum, it is plausible that insufficient levels of PD-L1 cause maternal $\beta$-cells to have greater susceptibility to cytotoxic damage, which can contribute to $\beta$-cell dysfunction. In GDM pregnancies, the low-grade inflammation that normally takes place in uncomplicated pregnancies is exacerbated (Hauguel-de Mouzon \& GuerreMillo 2006). The overexpression of pro-inflammatory cytokines contributes to excessive peripheral insulin resistance in the mother, necessitating enhanced insulin secretion to maintain euglycemia, which is often not met in GDM pregnancies (Lappas et al. 2005). Thus, it is plausible that a prolonged low-grade inflammatory environment persisting after parturition in the absence of the placenta could result from cytokines released by other organs, such as adipose tissue, which could contribute to $\beta$-cell dysfunction postpartum and lead to dysglycemia. Inflammation can contribute to $\beta$-cell dysfunction (Chen et al. 2015) via endoplasmic reticular stress resulting in decreased insulin sensitivity, as has been documented in GDM patients (Catalano 2014).

We investigated the changes that occur in mouse pancreas during pregnancy and found a three-fold increase in BCM on GD18, resulting largely from increased $\beta$-cell replication which peaked at GD12 (Beamish et al. 2017, Szlapinski et al. 2019). In our previous experiments, female offspring of mice fed a low protein (LP) diet during gestation and lactation were glucose intolerant at GD18 during their own pregnancy when compared to 
offspring from control diet-fed mothers (Szlapinski et al. 2019). Glucose intolerance was associated with reduced $\beta$-cell proliferation leading to a lower BCM, in addition to reduced glucose-stimulated insulin secretion (GSIS). Since GDM increases the risk of the mother developing subsequent T2DM, we have used the previously mentioned mouse model of gestational glucose intolerance to follow animals after parturition and determine whether glucose tolerance normalizes postpartum and what the longterm effects of glucose intolerance during pregnancy are on maternal pancreas morphometry. The mechanisms contributing to adaptive BCM expansion during pregnancy in rodents have been extensively studied. However, few studies exist investigating changes that occur past 7-10 days postpartum, and to our knowledge no data exists comparing pancreas morphometry after hyperglycemic and control pregnancy. We aimed to determine: (1) what happens to glucose tolerance and islet morphology postpartum after a normal vs a hyperglycemic pregnancy and (2) the possible involvement of cytokines and PD-L1 in long-term changes in islet morphology and glucose tolerance postpartum.

\section{Materials and methods}

\section{Animals and sample collection}

Adult (6-week-old) C57BL/6 male and female mice (Charles River) were housed in a temperature-controlled room with $12 \mathrm{~h}$ light:12 $\mathrm{h}$ darkness cycle at Lawson Health Research Institute (London, ON, Canada). Water and food were given ad libitum. Mice showing gestational glucose intolerance were generated using a previously described protocol involving a dietary insult during early life (Szlapinski et al. 2019). Briefly, timed pregnant F0 females were randomly allocated to either a control
(C, 20\% protein; Bio-Serv, Frenchtown, NJ, USA) or low protein diet (LP, 8\%) group, where an increase in carbohydrate in LP diet yields an isocalorific diet compared to control chow. F0 dams were fed either the LP or C diet throughout gestation and lactation. A total of $12 \mathrm{LP}$ and $12 \mathrm{C}$ litters were used. On postnatal day (PND) 21, female offspring (F1) were weaned onto C diet for the remainder of the study (Fig. 1). At maturity (PND42), female offspring (F1) of LP and C diet-fed mothers were randomly allocated into two subsequent study groups: pregnant (LPP, CP) or non-pregnant (LPNP, CNP). All pregnant-grouped females were mated with $\mathrm{C}$ diet-fed males, separated upon confirmation of pregnancy by vaginal plug and housed individually for the remainder of the experiment. Upon birth of pups, CP and LPP females were randomly allocated to one of three timepoints after parturition (postpartum day (PPD) 7, PPD30, or PPD90). For consistency, pups from all litters were euthanized at postnatal day 7. Mothers (F1) were euthanized by $\mathrm{CO}_{2}$ asphyxia following an intraperitoneal glucose tolerance test (IPGTT) on PPD7, PPD30, or PPD90 for comparison to non-pregnant agematched F1 females. Data (histology and IPGTT) for nonpregnant and GD18 animals, except for in vivo serum quantification, presented here have previously been published and are being used as a comparison to novel postpartum data in this study (Szlapinski et al. 2019). Following the IPGTT, maternal (F1) blood was collected via cardiac puncture in order to quantify serum insulin, glucagon, and PD-L1 quantified using an Ultra Sensitive Mouse Insulin ELISA kit (Crystal Chem, Downers Grove, IL, USA), Mouse Glucagon ELISA kit (Crystal Chem), and PD-L1 ELISA assay (LifeSpan Biosciences, Seattle, WA, USA), respectively. Data were collected using a BioRad iMark plate reader and analyzed using Microplate Manager Software.

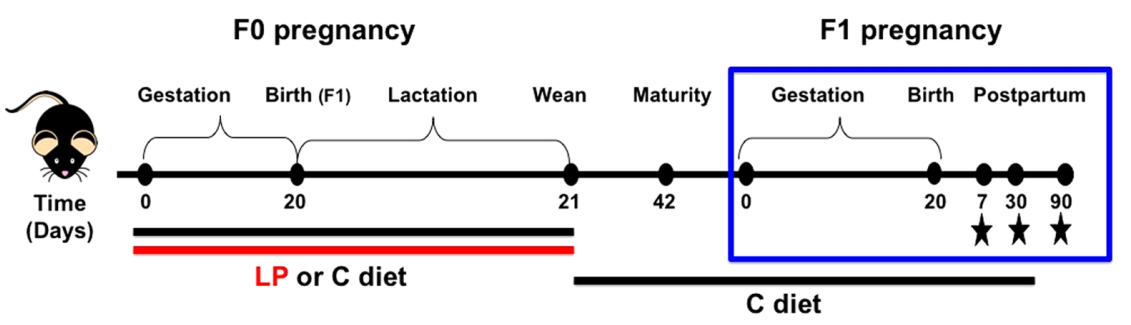

Figure 1

Experimental timeline for the generation of mice with gestational glucose intolerance and timepoints for sample collection. Female offspring (F1) were exposed to the low protein (LP) or control (C) diet during gestation and lactation and weaned onto C diet. At maturity, F1 females were mated with C-fed males. Pups (F2) from all litters were euthanized at postnatal day 7 . Stars demonstrate timepoints where an intraperitoneal glucose tolerance test was performed, the pancreas was removed for histology $(n=4-7$ animals for each group), and serum was collected via cardiac puncture. A full color version of this figure is available at https://doi.org/10.1530/ JOE-20-0012. 
Pancreata were removed immediately following euthanasia and fixed in 4\% paraformaldehyde for histology (Electron Microscopy Sciences, Hatfield, PA, USA). Fixed pancreas tissue was prepared and sectioned as previously described (Beamish et al. 2016). At least two $7 \mu \mathrm{m}$-thick cryosections (replicates) were cut from each pancreas with an interval between each section $>100 \mu \mathrm{m}$ representing at least two longitudinal slices through the pancreas. Sections included both the head and tail of the pancreas. All animal procedures were approved by the Animal Care Committee of Western University in accordance with the guidelines of the Canadian Council for Animal Care.

\section{Glucose tolerance tests}

An IPGTT was performed on all animals prior to euthanasia, as previously described (Chamson-Reig et al. 2009), at the assigned day postpartum or age for the agematched group ( $n=4-7 \mathrm{C}$ and LP animals per timepoint). Mice were fasted for $4 \mathrm{~h}$ with free access to water, injected intraperitoneally with $5 \mu \mathrm{L} / \mathrm{g}$ body weight of $40 \%$ glucose solution ( $2 \mathrm{~g} / \mathrm{kg}$ body weight glucose, Sigma Aldrich), and blood glucose measured from the tail at $0,5,15,30,60$, 90 , and 120 min using a One Touch Ultra2 glucometer.

\section{Immunohistochemistry and morphometric analysis}

Fluorescence immunohistochemistry was performed to localize insulin ( $\beta$-cells) and glucagon ( $\alpha$-cells) for morphometric analysis as previously described (Szlapinski et al. 2019). Every insulin and glucagon-expressing cell was imaged at $20 \times$ with the observer being blind to tissue identity using a Nikon Eclipse TS2R inverted microscope with NIS-Elements. The microscope was equipped with an LED light source with emission bandwidths set to $460 / 50 \mathrm{~nm}$ for blue emission, 535/50 $\mathrm{nm}$ for green emission, and $590 / 40 \mathrm{~nm}$ for red emission. For morphometric analyses, manual tracing of all islets for the tissue section was completed using ImageJ to quantitate fractional $\alpha$ - and $\beta$-cell area (sum of all glucagon or insulin-expressing areas divided by the whole pancreas surface area). Fractional areas were calculated from at least two sections (replicates) per pancreas ( $n=4-6 \mathrm{C}$ and LP animals per timepoint). Islets were counted per tissue section and further separated by size into small $\left(<5000 \mu \mathrm{m}^{2}\right)$, medium $\left(5000-10,000 \mu \mathrm{m}^{2}\right)$, or large $\left(>10,000 \mu \mathrm{m}^{2}\right)$ islets as previously described (Chamson-Reig et al. 2006). ApopTag Plus In Situ Apoptosis Fluorescein Detection Kit (S7111, EMD Millipore) was used to identify apoptotic $\beta$-cells using the terminal deoxynucleotidyl transferase dUTP nick end labeling (TUNEL) assay.

\section{Protein extraction and cytokine analysis}

Posterior s.c. white adipose tissue (27-50 mg) was lysed for $30 \mathrm{~min}$ in an ice-cold buffer as previously described (de Castro et al. 2011). Cytokine levels in adipose tissue protein extracts ( $n=3-4 \mathrm{C}$ and LP animals per timepoint, PPD90 C $n=2$ ) were determined by multiplexing in a Bioplex system using customized kits from R\&D systems (Magnetic Luminex assay) for cytokines of interest (TNF- $\alpha$, IL-6, IL-1 $\beta$, and IFN $\gamma$ ). Values were normalized to weight of sample.

\section{Statistical analysis}

Data are presented as mean \pm s.E.M., with statistics analyzed using GraphPad Prism (version 5.0). To determine effects of pregnancy on pancreas morphometry postpartum (CP), a one-way ANOVA was used followed by Bonferroni's post-hoc test. For comparisons of LPP and CP groups over time, a two-way ANOVA followed by Bonferroni's post-hoc test was used. A repeated measures two-way ANOVA followed by Bonferroni's post-hoc test was used for comparison of the IPGTT curves between treatment groups (LP vs C) at each timepoint after parturition. An unpaired two-tailed Student's $t$-test was used for comparison of IL-6 levels at PPD7 between dietary groups. Each animal presented as a single unit of analysis (n). Only the postpartum timepoints were included in statistical analyses to present novel data. To account for differences in litter sizes, the mean litter size for LPP and CP groups was determined. Litter sizes that were more than two standard deviations greater than the mean were considered outliers. This did not result in any outliers in the data set. Statistical significance was determined as $P<0.05$.

\section{Results}

\section{Mice with gestational glucose intolerance display} altered pregnancy characteristics

Offspring born to dams fed a LP diet during gestation and lactation (F1, LPP) weighed less than controls (F1, CP) throughout gestation (Fig. 2A) and gained significantly less weight than CP females at GD17 and 19 (Fig. 2B). There were no significant differences in body weight 
A

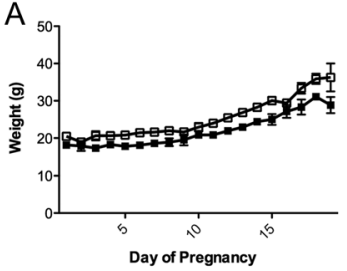

B

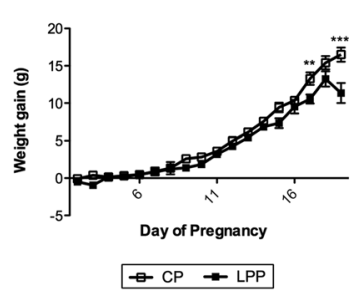

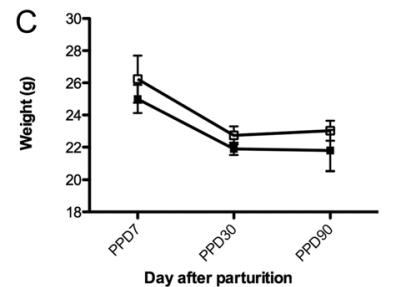

Figure 2

Mice with gestational glucose intolerance display altered pregnancy characteristics. (A) Pregnant female mice born to dams fed a low protein diet (LPP) weighed less and (B) gained less weight than pregnant female mice born to dams fed a control diet (CP) throughout pregnancy ( $n=7 \mathrm{CP}$ females, $n=10$ LPP females). (C) No differences in body weight were found after parturition between dietary groups ( $n=4-6 \mathrm{CP}$ and LPP females). $\star \star \star P<0.001, * \star P<0.01$

between dietary groups after parturition (Fig. 2C). Body weight differed with time after parturition in both dietary groups $(P<0.01$, Fig. 2C).

\section{Mice with gestational glucose intolerance demonstrate prolonged glucose intolerance after parturition}

We compared glucose tolerance in LPP vs CP mice after parturition. LPP females that were relatively glucose intolerant at GD18 continued to display glucose intolerance at PPD7 with higher blood glucose levels at 15 and 30 min into an IPGTT (Fig. 3A). The relative glucose intolerance persisted at 1 month postpartum (PPD30, Fig. 3B). However, by 3 months postpartum (PPD90, Fig. 3C) LPP females had a similar glycemic profile
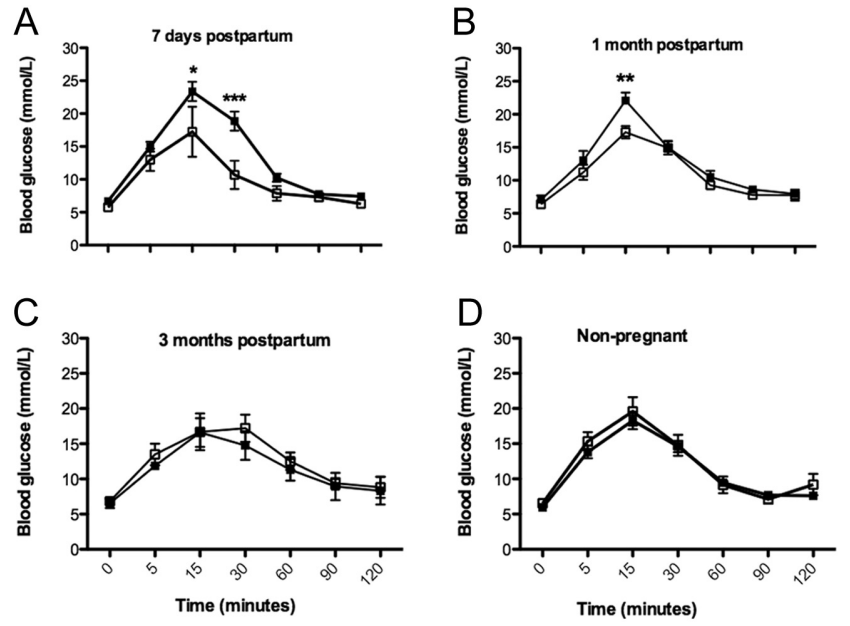

$\rightarrow C P \rightarrow$ LPP

Figure 3

LPP females show prolonged glucose intolerance after parturition. Pregnant female mice born to dams fed a low protein diet (LPP) show glucose intolerance relative to pregnant female mice born to dams fed a control diet (CP) at (A) postpartum day 7 (PPD7, $n=6$ CP females, 7 LPP females) and continue to be glucose intolerant at (B) 1 month postpartum (PPD30, $n=6$ CP females, 5 LPP females). (C) However, by 3 months postpartum ( $n=4$ CP females, 4 LPP females) LPP females display a glycemic profile similar to that of a control and demonstrate a similar glycemic profile to that of a (D) non-pregnant animal ( $n=6 \mathrm{C}$ nonpregnant females, 5 LP non-pregnant females). ${ }^{*} * P<0.001, * \star P<0.01$, $\star p<0.05$. as that seen in controls. Furthermore, the glycemic profile at PPD90 was restored to that of a non-pregnant animal (Fig. 3D). There were no significant differences in fasting blood glucose levels between dietary groups or with time (Supplementary Fig. 1A, see section on supplementary materials given at the end of this article). Additionally, area under the glucose tolerance curve was higher in the LPP group at PPD7 compared to controls (Supplementary Fig. 1B).

\section{Pregnancy alters pancreas morphology postpartum after both control and hyperglycemic pregnancies}

We investigated how endocrine pancreas morphology changed after parturition, both in CP and LPP groups. Pancreatic sections were immunostained for insulin ( $\beta$-cells) and glucagon ( $\alpha$-cells) for morphometric analyses (Fig. 4). Since pancreas weight was relatively higher in the LPP group at PPD30 and PPD90 (Fig. 5A),
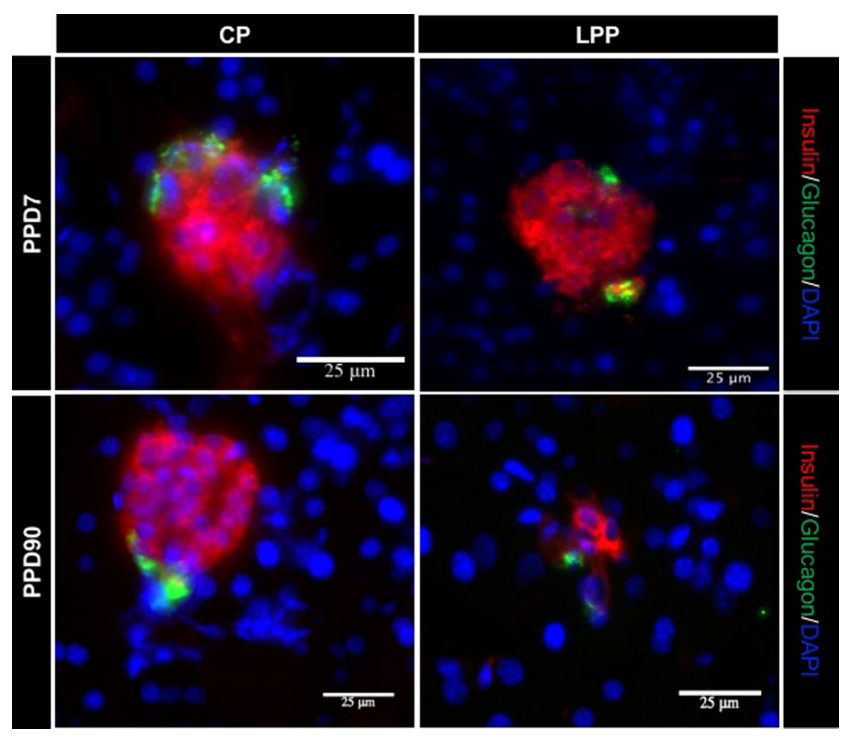

\section{Figure 4}

Representative immunofluorescent images demonstrating $\beta$ - (insulin, red) and $\alpha$-cells (glucagon, green) in pregnant female mice born to dams fed a control diet (CP) and pregnant female mice born to dams fed a low protein diet (LPP) at postpartum days (PPD) 7 and PPD90. Nuclei were counterstained with DAPI (blue). Scale bar represents 25 microns. 

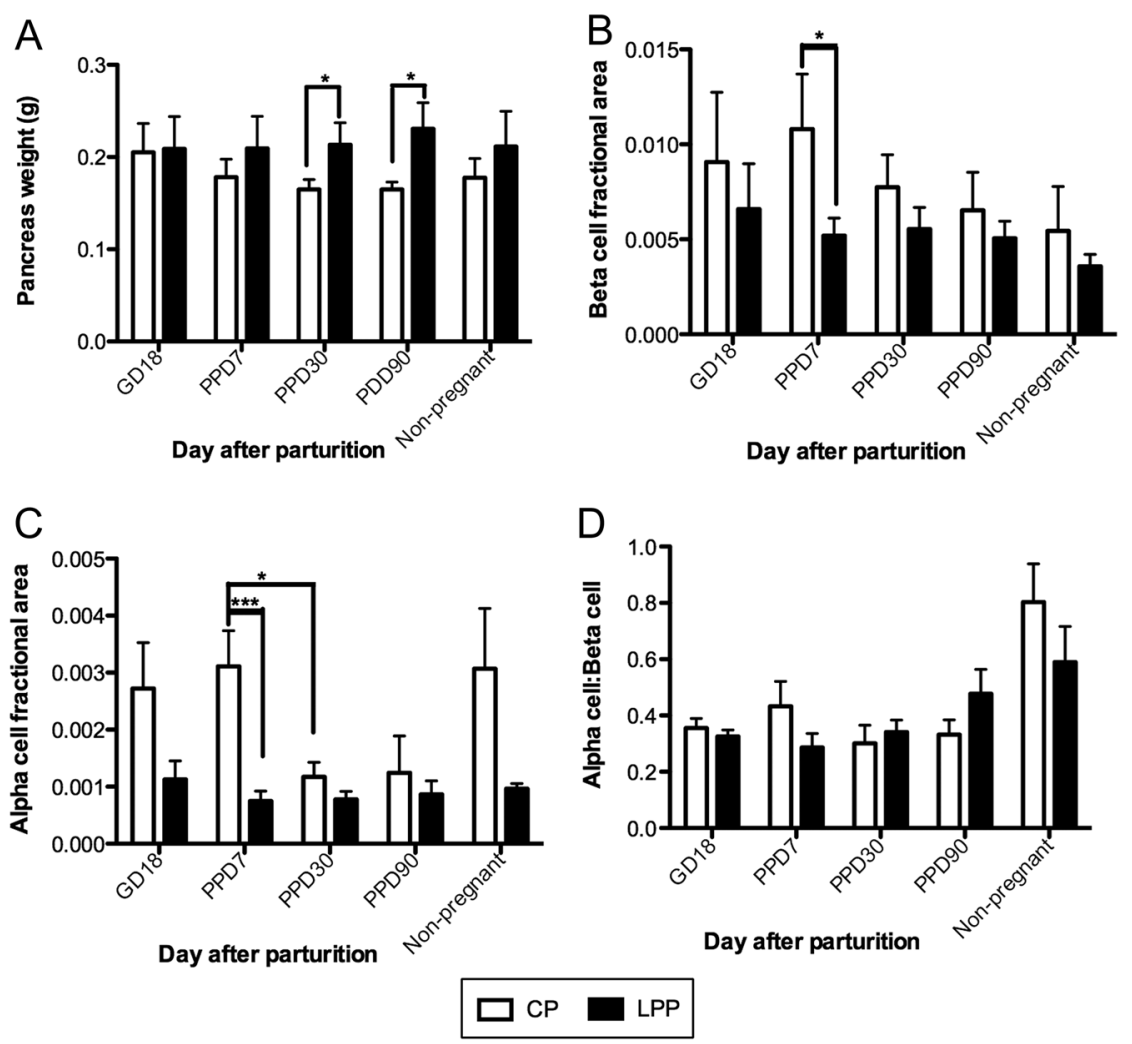

\begin{abstract}
Figure 5
Pregnancy alters pancreas morphology postpartum after control and hyperglycemic pregnancies. (A) Pancreas weight was higher in pregnant female mice born to dams fed a low protein diet (LPP) at postpartum day (PPD) 30 and PPD90 relative to pregnant female mice born to dams fed a control diet (CP). Both the fractional (B) $\beta$-cell area and (C) $\alpha$-cell area were lower in LPP animals vs controls at PPD7. Furthermore, $\alpha$-cell area was lower at PPD30 vs PPD7 in CP females. (D) Alpha to $\beta$-cell ratio did not differ between dietary groups or timepoints after parturition ( $n=4-6$ CP and LPP females). $* * * P<0.001$, $\star P<0.05$.
\end{abstract}

$\beta / \alpha$-cell fractional areas were used for histological analyses rather than BCM. Beta-cell fractional area was lower in LPP animals compared to CP animals at PPD7 (Fig. 5B). Notably, relative to PPD7, CP females had a $\sim 30 \%$ reduction in $\beta$-cell fractional area at PPD30 and a $\sim 40 \%$ reduction by PPD90 (Fig. 5B), the latter resulting in a $\beta$-cell fractional area comparable to a non-pregnant animal. In contrast to CP females, $\beta$-cell fractional area did not further decrease postpartum in the LPP group, rather the values remained at a similar level from GD18 up until PPD90 (Fig. 5B). Alpha-cell fractional area was lower in LPP animals compared to CP at PPD7 (Fig. 5C). Furthermore, $\alpha$-cell area was lower at PPD30 vs PPD7 in CP females. There were no significant differences in the $\alpha$ - to $\beta$-cell ratio (Fig. 5D) between dietary groups or with time after parturition. No evidence of dual-stained insulin/TUNEL cells was found when investigating the contribution of $\beta$-cell apoptosis at PPD7.

Islet quantification demonstrated that the number of small, medium, and large-sized islets varied with time after parturition (Table 1). At PPD90, there were significantly more small-sized islets in the LPP females compared to

Table 1 Islet size distributions are altered postpartum after control and hyperglycemic pregnancies in mice.

\begin{tabular}{|c|c|c|c|c|c|}
\hline Timepoint & Treatment & Small $\left(5000 \mu \mathrm{m}^{2}\right)$ & Medium $\left(5000-10,000 \mu \mathrm{m}^{2}\right)$ & Large $\left(10,000 \mu \mathrm{m}^{2}\right)$ & Mean islet size $\left(\mu \mathrm{m}^{2}\right)$ \\
\hline \multirow[t]{2}{*}{ Non-pregnant } & Control & 9.64 & 1.79 & 2.00 & 4974 \\
\hline & LP & 7.50 & 1.42 & 1.33 & 4076 \\
\hline \multirow[t]{2}{*}{ GD18 } & $\mathrm{CP}$ & 17.88 & 3.00 & 5.00 & 7967 \\
\hline & LPP & 7.90 & 1.30 & 1.60 & 4324 \\
\hline \multirow[t]{2}{*}{ PPD7 } & $\mathrm{CP}$ & 13.92 & 2.50 & 3.83 & 6394 \\
\hline & LPP & 11.80 & 1.30 & 1.80 & 3599 \\
\hline \multirow[t]{2}{*}{ PPD30 } & $\mathrm{CP}$ & 17.20 & 4.50 & 6.70 & 6141 \\
\hline & LPP & 13.73 & 2.63 & 4.00 & 5390 \\
\hline \multirow[t]{2}{*}{ PPD90 } & $\mathrm{CP}$ & 5.29 & 1.50 & 2.13 & 6403 \\
\hline & LPP & $12.29 a$ & 2.00 & 2.00 & 6373 \\
\hline
\end{tabular}

The number of small, medium, and large-sized islets varied with time after parturition in both dietary groups $(P<0.05)$. There were more small islets at postpartum day (PPD) 90 in pregnant female mice born to dams fed a low protein diet (LPP) compared to pregnant female mice born to dams fed a control diet (CP). There were no significant differences in mean islet size after parturition between dietary groups. aP $<0.05$ LPP vs CP. 
controls (Table 1). There were no significant differences in mean islet size after parturition between dietary groups (Table 1). However, the mean islet size was 1.8-fold higher at PPD90 $\left(6373 \pm 2065 \mu \mathrm{m}^{2}\right)$ compared to PPD7 $\left(3599 \pm 452 \mu \mathrm{m}^{2}\right)$ in the LPP group $(P=0.147$, Table 1$)$.

\section{Cytokines may contribute to glucose intolerance after parturition in mice experiencing hyperglycemic pregnancies}

To investigate a potential contribution to prolonged glucose intolerance after parturition in the LPP group, we measured levels of representative cytokines in adipose tissue extracts by determining levels of TNF- $\alpha$, IL- 6 , IL-1 $\beta$, and IFN $\gamma$. TNF- $\alpha$ levels varied with time after parturition in both dietary groups and were 1.4-fold higher in the LPP group at PPD7 compared to controls $(3.56 \pm 1.10 \mathrm{pg} / \mathrm{mL} / \mathrm{mg}$ tissue vs $2.55 \pm 0.98 \mathrm{pg} / \mathrm{mL} / \mathrm{mg}$ tissue, $P=0.534$, Fig. 6 A). Levels of IL-6 were higher in the LPP group at PPD7 compared to controls (Fig. 6B). There were no significant differences in levels of IL-1 $\beta$ with time after parturition or between dietary groups (Fig. 6C). However, IL-1 $\beta$ was 1.4-fold higher at PPD7 in LPP compared to controls $(698.5 \pm 254.7 \mathrm{pg} / \mathrm{mL} / \mathrm{mg}$ tissue vs $517.8 \pm 274.2 \mathrm{pg} / \mathrm{mL} / \mathrm{mg}$ tissue, $P=0.654$, Fig. 6C). Levels of IFN $\gamma$ were 3.1fold higher at PPD7 in LPP compared to controls $(68.63 \pm 24.94 \mathrm{pg} / \mathrm{mL} / \mathrm{mg}$ tissue vs $21.88 \pm 4.51 \mathrm{pg} / \mathrm{mL} / \mathrm{mg}$ tissue, $P=0.139$, (Fig. 6D).
Next, circulating levels of PD-L1, insulin, and glucagon in serum were quantified. There were no significant differences in levels of PD-L1 between dietary groups. However, values differed with time after parturition, as levels of PD-L1 were higher at PPD90 compared to PPD7 in controls, but not in LPP mice (Fig. $7 \mathrm{~A})$. Of note, there was also a 2.7 -fold higher amount of PD-L1 present in LPP animals at PPD7 compared to controls $(145 \pm 50 \mathrm{pg} / \mathrm{mL}$ vs $55 \pm 29 \mathrm{pg} / \mathrm{mL}, P=0.214$, Fig. 7A). The ratio of serum insulin to glucagon varied with time after parturition in both CP and LPP mice (Fig. $7 \mathrm{~B})$, with a trend toward a higher ratio in the LPP group $(P=0.071$ LP vs C, Fig. 7B).

\section{Discussion}

GDM increases the subsequent risk of maternal dysglycemia or T2DM by up to 7-fold (Melchior et al. 2017), although the relationship of this risk to longstanding changes in $\beta$-cell histology postpartum is unknown. Using a mouse model of gestational glucose intolerance, we addressed this knowledge gap by showing that dams continue to show glucose intolerance for at least a month after parturition (PPD30), and that glycemic control did not normalize until 3 months postpartum (PPD90). This persistent glucose intolerance was associated with lower $\beta$ - and $\alpha$-cell fractional areas at PPD7 compared with control pregnancies representing an extension of the
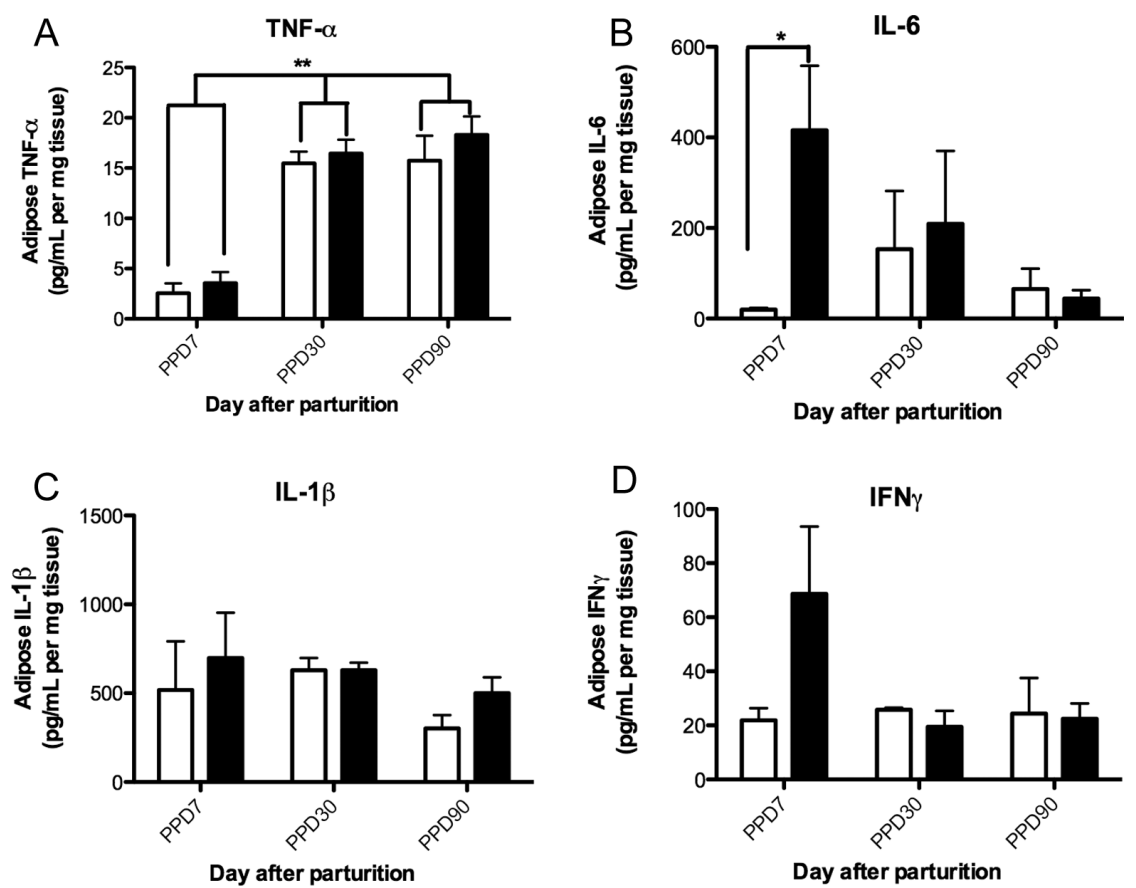

\section{Figure 6}

Altered cytokines may contribute to glucose intolerance in hyperglycemic mice after parturition. Adipose tissue content of: (A) TNF- $\alpha$, (B) IL-6, (C) IL-1 $\beta$, and (D) IFN $\gamma$ for pregnant female mice born to dams fed a control diet (CP) and pregnant female mice born to dams fed a low protein diet (LPP) at postpartum days (PPD) 7, 30, and 90. (A) TNF- $\alpha$ content significantly increased with time postpartum but not between dietary groups. (B) IL-6 content decreased with time but was significantly higher in LPP animals. (C) IL-1 $\beta$ and (D) IFN $\gamma$ content did not differ with time or between diets. $n=3-4, * * P<0.01, * P<0.05$. 

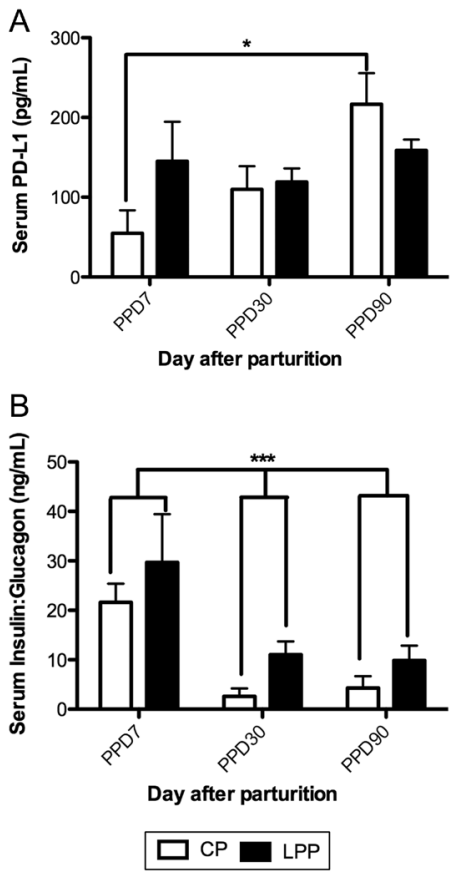

\section{Figure 7}

Pregnancy results in long-term alterations in serum levels of PD-L1, insulin, and glucagon after parturition. Serum levels of (A) PD-L1 and (B) insulin:glucagon ratio. (A) PD-L1 levels increased with time in pregnant female mice born to dams fed a control diet (CP) but not in pregnant female mice born to dams fed a low protein diet (LPP). (B) The serum insulin:glucagon ratio trended to be higher in LPP animals compared to controls $(P=0.0705)$ and varied with time in both groups. $n=3-5$, $* * * P<0.001, * P<0.05$

relative differences seen in late pregnancy for the glucoseintolerant dams.

Several studies have reported the presence of $\beta$-cell dysfunction postpartum clinically after GDM (Buchanan \& Xiang 2005, Retnakaran et al. 2008, Xiang et al. 2010, Molęda et al. 2013). Progressive $\beta$-cell dysfunction is likely the predominant factor that drives the transition from impaired glucose tolerance to T2DM after GDM (Bellamy et al. 2009, Saisho et al. 2013). In this study, LPP animals displayed glucose intolerance at PPD7 and PPD30 relative to controls. We have previously shown that LPP animals exhibit $\beta$-cell dysfunction at late gestation (GD18), resulting in reduced GSIS (Szlapinski et al. 2019). A previous clinical study classified $\beta$-cell dysfunction as the key factor in the development of postpartum dysglycemia among non-obese patients (Li et al. 2014); insulin resistance, however, was determined to be the driver of postpartum hyperglycemia in obese patients. Our animal model of gestational glucose intolerance, which represents the nonobese category, therefore accurately reflects the changes that occur during $\beta$-cell dysfunction after GDM, rather than another underlying cause (such as insulin resistance as seen in obese patients). Although pre-gestational obesity is a major driver of GDM, $20-30 \%$ of women that develop GDM do not fall into this category. Therefore, this implicates dysfunction at the level of the $\beta$-cell to GDM pathophysiology (Petra et al. 2019). This study thus proves useful for revealing underlying mechanisms of glucose intolerance postpartum characterized by $\beta$-cell dysfunction. Since the animal model represents only a mild hyperglycemia, the animals in this study were able to normalize blood glucose levels by PPD90. Nonetheless, it is plausible that additional metabolic stress such as a second pregnancy, or an age-related decline in $\beta$-cell function (Westacott et al. 2017), could precipitate T2DM.

Human studies of impaired glucose tolerance postpartum do not allow visualization of histological changes occurring in endocrine pancreas. Previous studies of healthy rodent pregnancy (Rieck \& Kaestner 2010, Beamish et al. 2017) report that offspring born to dams fed a control diet (CP) had a $\sim 50 \%$ reduction in BCM at PPD7 compared to GD18. In this study, CP females had a 30\% reduction in $\beta$-cell fractional area at PPD30 compared to PPD7 and $\sim 40 \%$ by PPD90 relative to PPD7, reducing $\beta$-cell fractional area to a level comparable to a nonpregnant animal. These differences could be attributed to the use of fractional area in this study instead of BCM. We used fractional area to exclude the effect of changes in exocrine tissue mass postpartum. Prolactin can alter exocrine tissue mass during lactation in mice (Matsuda et al. 1994), and we found differences in pancreatic weight postpartum in LPP mice. Thus, the use of fractional area provides a more accurate representation of endocrine changes in this study. These data support previous findings that $\beta$-cell apoptosis is occurring in controls after parturition in order to facilitate normalisation of BCM. This occurs, in part, due to a switch of $\beta$-cell serotonin receptor expression from $H T R 2 B$ to HTR $1 D$, mediating an inhibitory signal and promoting regression of $\mathrm{BCM}$ via increased $\beta$-cell apoptosis (Scaglia et al. 1995, Kim et al. 2010), in addition to higher levels of steroid hormones at late gestation which block lactogen-induced $\beta$-cell replication (Sorenson et al. 1993). Insulin/TUNEL staining was negative when investigating the contribution of $\beta$-cell apoptosis at PPD7, suggesting that the apoptotic processes likely occur earlier postpartum since BCM was already reduced by PPD7. Nonetheless, a potential explanation for the retained $\beta$-cell fractional area at PPD7 in controls could be due to persistently higher expression of serotonin synthetic enzyme tryptophan hydroxylase-1, Tph1, which normally increases during pregnancy in $\beta$-cells in order to mediate BCM expansion. A previous study found that 
Tph1 expression remained high at PPD7 until the end of lactation (PPD21) when levels returned to pre-pregnancy levels (Kim et al. 2010).

In terms of changes in pancreatic $\alpha$-cell abundance, fractional area was lower at PPD30 compared to PPD7 in CP females. Thus, it is evident that there is a prolonged effect of pregnancy on the ontogeny of $\alpha$-cells, as fractional area decreases to a level lower than the non-pregnant control at PPD90. These findings prompt interesting questions for future studies, especially concerning a second pregnancy and whether the pool of $\alpha$-cells would increase and expand as observed on GD18 in a healthy pregnancy? (QuesadaCandela et al. 2019, Szlapinski et al. 2019). Furthermore, would the pool of $\alpha$-cells take longer to replenish or is the $\alpha$-cell complement fully depleted after a first pregnancy?

In contrast to $\mathrm{CP}$ females, $\beta$-cell fractional area was not further decreased postpartum in LPP mice; the values instead remained at a similar level from GD18 to PPD90. There were no differences in $\beta$-cell proliferation between dietary groups at PPD7 compared to nonpregnant animals (Supplementary Fig. 1C). These data confirm previous findings that lactogen-induced $\beta$-cell proliferation that normally occurs during pregnancy, which is also functionally linked to increased levels of progesterone and estradiol as pregnancy progresses (Sorenson et al. 1993), is arrested by PPD7. However, since levels of $\beta$-cell replication were comparable in CP and LPP groups postpartum, this excludes $\beta$-cell replication as a mechanism for the sustained elevated fractional $\beta$-cell in LPP mice. As previously mentioned, we found no evidence of $\beta$-cell apoptosis at PPD7. These findings support clinical data that markers of $\beta$-cell loss were reduced in serum samples from women postpartum after GDM and reached levels seen in non-pregnant women (Kenna et al. 2016). Thus, our study is the first to provide histological evidence to support these clinical findings by demonstrating that less $\beta$-cell loss is occurring postpartum after mild GDM.

When comparing healthy and hyperglycemic pregnancies, both $\alpha$ - and $\beta$-cell fractional areas were lower in LPP mice at PPD7; a likely result of the insufficient endocrine pancreas adaptation previously found to occur at GD18, appearing to persist at PPD7. Importantly, $\alpha$-cells have been identified as a target for serotonin action, as a study in human islets showed that $\beta$-cell-derived serotonin inhibited glucagon secretion in response to high glucose (Almaça et al. 2016). Due to a reduced fractional $\beta$-cell area in LPP animals at PPD7 in this study, it is plausible that $\alpha$-cells receive a reduced serotonergic input from $\beta$-cells and thus lose their ability to regulate glucagon secretion. This may result in uncontrolled glucagon secretion and could contribute to the hyperglycemia seen in LPP animals at PPD7. This was shown to occur in vivo in women with GDM through a lack of suppression of plasma glucagon at late pregnancy that persisted postpartum (Beis et al. 2005). These findings highlight an important role for pancreatic $\alpha$-cells in pregnancy which has previously been overlooked.

Despite the lack of a placental hormone stimulus to endocrine cell expansion postnatally, the $\beta$-cell fractional area did not change in LPP mice. This might be a compensatory mechanism in attempt to attain euglycemia. Size stratification of islets varied at PPD7 and PPD30 in both control and LP groups, but there were no differences between dietary groups. This suggests that pregnancy itself causes re-modeling of islet populations after parturition. Additionally, there were more small islets in LPP females compared to controls found at PPD90, contributing to a recovered mean islet size comparable to that of a control. Thus, these data support an additional adaptive response in endocrine cells postpartum in LPP animals. These data could implicate $\beta$-cell neogenesis of small islets to facilitate normalization of mean islet size at PPD90 as a compensatory mechanism, thereby resulting in a rescue of glucose tolerance relative to controls. The LPP group had a higher ratio of insulin relative to glucagon after parturition compared to controls, which provides further support of such an adaptive response.

Pro-inflammatory cytokines can modify insulin signaling pathways and can lead to $\beta$-cell dysfunction (Kolb $\&$ Mandrup-Poulsen 2005). Consequently, overexpression of cytokines can accelerate inflammation and exacerbate insulin resistance. Women with GDM have been shown to have increased circulating levels of the pro-inflammatory cytokines TNF- $\alpha$, IL-1 $\beta$, and IL- 6 , all of which are associated with $\beta$-cell dysfunction (Yang et al. 2018, Petra et al. 2019). Thus, it is plausible that prolonged glucose intolerance or T2DM after GDM could involve persistent inflammation postpartum. Both mouse and human placenta express multiple cytokines that contribute to the state of insulin resistance that occurs during pregnancy; for instance, TNF- $\alpha$ induces IRS-1 serine phosphorylation, which contributes to BCM expansion and insulin resistance in pregnancy (Kirwan et al. 2002, Barbour et al. 2007). In women with GDM, the decrease in insulin receptor tyrosine kinase phosphorylation does not improve postpartum as it does in women following a healthy pregnancy (Kirwan et al. 2004, Catalano 2014). In the present model, levels of TNF- $\alpha$ were relatively higher in the LPP animals compared to controls after parturition which could contribute, in part, to the glucose intolerance seen at PPD7 and PPD30. 
TNF- $\alpha$ levels in adipose increased with time, as seen in non-obese women in a study investigating longitudinal changes in serum pro-inflammatory markers across pregnancy and postpartum (Christian \& Porter 2014). IL-6 has been found to be significantly higher in women with GDM, independent of adiposity (Mirghani Dirar \& Doupis 2017), as observed in adipose samples from the non-obese animals used in this study where levels of IL-6 were higher in LPP mice at PPD7 compared to controls. Interestingly, IL-6 levels were reduced in both $\mathrm{CP}$ and LPP groups by PPD90, concomitant with the return of glucose tolerance to control values. Some studies have found that high concentrations of IL- 6 could promote $\beta$-cell apoptosis and contribute to glucose intolerance (Jin et al. 2016). However, since $\beta$-cell area did not change at any time point after parturition in LPP mice, it is more likely that IL-6 is exerting effects on non-islet tissues. IL-6 has been shown to increase lipolysis in adipocytes, damage mitochondria and GLUT2 function, and as a result decrease insulin sensitivity (Dessein et al. 2002). It is reasonable that higher IL-6 tissue levels contibuted to increased insulin resistance postpartum in LPP mice (Barbour et al. 2007). IL-6 can also induce production of IL-1 $\beta$ and TNF- $\alpha$ (Calabrese \& Rose-John 2014), further intensifying levels of pro-inflammatory cytokines postpartum and contributing to $\beta$-cell dysfunction and glucose intolerance. However, IL-1 $\beta$ levels in adipose tissue did not differ between dietary groups in the present studies. Collectively, these findings implicate a potential contribution of inflammation to insulin resistance postpartum in LPP mice, resulting in a maintaned $\beta$-cell area resistant to apoptosis postpartum.

An increase in cytokine production occurs in healthy pregnancies. However, the activity of PD-L1 also increases and attenuates the low-grade inflammatory immune response (Meggyes et al. 2019), potentially protecting maternal $\beta$-cells from cytotoxic damage. In the present study, levels of PD-L1 were relatively higher in LPP mice at PPD7 compared to controls. This may have helped to enhance $\beta$-cell survival in the face of a higher cytokine environment, as has been observed in autoimmune diabetes in NOD mice (Wang et al. 2008). The PD-L1 ligand has also been shown to be expressed in $\beta$-cells of individuals with type 1 diabetes as a possible attempt to attenuate autoimmune attack (Colli et al. 2018). However, PD-L1 was absent from islets of non-diabetic controls. Furthermore, the same study showed that IFN $\gamma$ induced PD-L1 mRNA expression in human pancreatic $\beta$-cells in vitro, potentially implicating IFN $\gamma$ at PPD7 in LPP mice in this study as a mechanism for increasing PD-L1 levels. This pathway might also mediate $\beta$-cell neogenesis leading to a subsequent increase in the number of small islets observed at PPD90. This inflammatory pathway may also explain why $\beta$-cell fractional area remained elevated in LPP mice postpartum. Follow-up experiments treating LPP and CP mice at PPD7/30/90 with a PD-L1 inhibitor would be insightful to investigate potential differences in the ratio of serum insulin to glucagon. Interestingly, PD-L1 levels increased from PPD7 to PPD90 in controls. As a multitude of immunological changes involving both pro- and anti-inflmamatory cytokines are occuring throughout pregnancy to the postpartum period, it is plasuble that serum PD-L1 levels were elevated in controls, in part, to suppress maternal immunity or to mediate a protective effect on $\beta$-cells against higher levels of TNF- $\alpha$ at postpartum. Interestingly, since PD-L1 levels were 25\% lower in LPP mice at PPD90, perhaps these animals will be more prone to cytoxicic $\beta$-cell damage and could be on the trajectory to dysglycemia or T2DM. PD-L1 has also been identified as a biomarker for GDM in humans (Ye et al. 2017) and our findings support a potential role as a marker for prolonged glucose intolerance after GDM.

In summary, we present novel findings of the ontogeny of $\alpha$ - and $\beta$-cell fractional areas of islet morphology and glucose tolerance postpartum in normal and hyperglycemic mouse pregnancies. The results demonstrate long-term pancreatic re-modeling after parturition involving both pancreatic $\alpha$ - and $\beta$-cells, which was associated with changes in the pro-inflammatory environment. These findings are informative in understanding the pathophysiology involved in the progression from GDM to glucose intolerance and T2DM.

\section{Supplementary materials}

This is linked to the online version of the paper at https://doi.org/10.1530/ JOE-20-0012.

\section{Declaration of interest}

The authors declare that there is no conflict of interest that could be perceived as prejudicing the impartiality of the research reported.

\section{Funding}

This work was supported by grants from Lawson Foundation (St Joseph's Health Care Foundation), London, ON, Canada. S S is a recipient of the Ontario Graduate Scholarship.

\section{Author contribution statement}

S S and D H designed the experiments. D H provided resources, supervision and acquired funding. $S \mathrm{~S}$ performed data curation and conducted the research in cooperation with $A B, S D, R K, G R$, and B S. S S and 
D H analyzed and interpreted the data. SS wrote the original draft. All authors participated in the revision of the manuscript and approved the final version of the manuscript.

\section{Acknowledgements}

The authors thank Dr Edith Arany for guidance with manuscript preparation and Dr Julio Sevillano and Shannon Seney for technical assistance with the Luminex Multiplex experiment.

\section{References}

Almaça J, Molina J, Menegaz D, Pronin AN, Tamayo A, Slepak V, Berggren PO \& Caicedo A 2016 Human beta cells produce and release serotonin to inhibit glucagon secretion from alpha cells. Cell Reports 17 3281-3291. (https://doi.org/10.1016/j.celrep.2016.11.072)

Barbour LA, McCurdy CE, Hernandez TL, Kirwan JP, Catalano PM \& Friedman JE 2007 Cellular mechanisms for insulin resistance in normal pregnancy and gestational diabetes. Diabetes Care 30 (Supplement 2) S112-S119. (https://doi.org/10.2337/dc07-s202)

Beamish CA, Strutt BJ, Arany EJ \& Hill DJ 2016 Insulin-positive, Glut2low cells present within mouse pancreas exhibit lineage plasticity and are enriched within extra-islet endocrine cell clusters. Islets 8 65-82. (https://doi.org/10.1080/19382014.2016.1162367)

Beamish CA, Zhang L, Szlapinski SK, Strutt BJ \& Hill DJ 2017 An increase in immature $\beta$-cells lacking Glut2 precedes the expansion of $\beta$-cell mass in the pregnant mouse. PLOS ONE 12 e0182256. (https://doi. org/10.1371/journal.pone.0182256)

Beis C, Grigorakis SI, Philippou G, Alevizaki M \& Anastasiou E 2005 Lack of suppression of plasma glucagon levels in late pregnancy persists postpartum only in women with previous gestational diabetes mellitus. Acta Diabetologica 42 31-35. (https://doi.org/10.1007/ s00592-005-0171-5)

Bellamy L, Casas JP, Hingorani AD \& Williams D 2009 Type 2 diabetes mellitus after gestational diabetes: a systematic review and metaanalysis. Lancet 373 1773-1779. (https://doi.org/10.1016/S01406736(09)60731-5)

Buchanan TA \& Xiang AH 2005 Gestational diabetes mellitus. Journal of Clinical Investigation 115 485-491. (https://doi.org/10.1172/JCI24531)

Butler AE, Cao-Minh L, Galasso R, Rizza RA, Corradin A, Cobelli C \& Butler PC 2010 Adaptive changes in pancreatic beta cell fractional area and beta cell turnover in human pregnancy. Diabetologia $\mathbf{5 3}$ 2167-2176. (https://doi.org/10.1007/s00125-010-1809-6)

Calabrese LH \& Rose-John S 2014 IL-6 biology: implications for clinical targeting in rheumatic disease. Nature Reviews: Rheumatology $\mathbf{1 0}$ 720-727. (https://doi.org/10.1038/nrrheum.2014.127)

Catalano PM 2014 Trying to understand gestational diabetes. Diabetic Medicine 31 273-281. (https://doi.org/10.1111/dme.12381)

Chamson-Reig A, Thyssen SM, Arany E \& Hill DJ 2006 Altered pancreatic morphology in the offspring of pregnant rats given reduced dietary protein is time and gender specific. Journal of Endocrinology 191 83-92. (https://doi.org/10.1677/joe.1.06754)

Chamson-Reig A, Thyssen SM, Hill DJ \& Arany E 2009 Exposure of the pregnant rat to low protein diet causes impaired glucose homeostasis in the young adult offspring by different mechanisms in males and females. Experimental Biology and Medicine 234 1425-1436. (https:// doi.org/10.3181/0902-RM-69)

Chen L, Chen R, Wang H \& Liang F 2015 Mechanisms linking inflammation to insulin resistance. International Journal of Endocrinology 2015 508409. (https://doi.org/10.1155/2015/508409)

Christian LM \& Porter K 2014 Longitudinal changes in serum proinflammatory markers across pregnancy and postpartum: effects of maternal body mass index. Cytokine 70 134-140. (https://doi. org/10.1016/j.cyto.2014.06.018) (c) 2020 Society for Endocrinology Published by Bioscientifica Ltd. Printed in Great Britain
Colli ML, Hill JLE, Marroquí L, Chaffey J, Dos Santos RS, Leete P, Coomans de Brachène A, Paula FMM, Op de Beeck A, Castela A, et al. 2018 PDL1 is expressed in the islets of people with type 1 diabetes and is up-regulated by interferons- $\alpha$ and- $\gamma$ via IRF1 induction. EBioMedicine 36 367-375. (https://doi.org/10.1016/j. ebiom.2018.09.040)

de Castro J, Sevillano J, Marciniak J, Rodriguez R, González-Martín C, Viana M, Eun-suk OH, de Mouzon SH, Herrera E \& Ramos MP 2011 Implication of low level inflammation in the insulin resistance of adipose tissue at late pregnancy. Endocrinology 152 4094-4105. (https://doi.org/10.1210/en.2011-0068)

Dessein PH, Joffe BI \& Stanwix AE 2002 Effects of disease modifying agents and dietary intervention on insulin resistance and dyslipidemia in inflammatory arthritis: a pilot study. Arthritis Research 4 R12. (https://doi.org/10.1186/ar597)

Ferrara A 2007 Increasing prevalence of gestational diabetes mellitus: a public health perspective. Diabetes Care 30 (Supplement 2) S141-S146. (https://doi.org/10.2337/dc07-s206)

Garcia-Vargas L, Addison SS, Nistala R, Kurukulasuriya D \& Sowers JR 2012 Gestational diabetes and the offspring: implications in the development of the cardiorenal metabolic syndrome in offspring. Cardiorenal Medicine 2 134-142. (https://doi.org/10.1159/000337734)

Gibbs RS, Romero R, Hillier SL, Eschenbach DA \& Sweet RL 1992 A review of premature birth and subclinical infection. American Journal of Obstetrics and Gynecology 166 1515-1528. (https://doi. org/10.1016/0002-9378(92)91628-N)

Hauguel-de Mouzon S \& Guerre-Millo M 2006 The placenta cytokine network and inflammatory signals. Placenta 27 794-798. (https://doi. org/10.1016/j.placenta.2005.08.009)

Heida KY, Franx A, van Rijn BB, Eijkemans MJC, Boer JMA, Verschuren MWM, Oudijk MA, Bots ML \& van der Schouw YT 2015 Earlier age of onset of chronic hypertension and type 2 diabetes mellitus after a hypertensive disorder of pregnancy or gestational diabetes mellitus. Hypertension 66 1116-1122. (https://doi. org/10.1161/HYPERTENSIONAHA.115.06005)

Jin H, Ning Y, Zhou H \& Wang Y 2016 IL-6 promotes islet $\beta$-cel dysfunction in rat collagen-induced arthritis. Journal of Diabetes Research 2016 7592931. (https://doi.org/10.1155/2016/7592931)

Kenna LA, Olsen JA, Spelios MG, Radin MS \& Akirav EM 2016 -Cell death is decreased in women with gestational diabetes mellitus. Diabetology and Metabolic Syndrome 8 60. (https://doi.org/10.1186/ s13098-016-0175-z)

Kim H, Toyofuku Y, Lynn FC, Chak E, Uchida T, Mizukami H, Fujitani Y, Kawamori R, Miyatsuka T, Kosaka Y, et al. 2010 Serotonin regulates pancreatic beta cell mass during pregnancy. Nature Medicine $\mathbf{1 6}$ 804-808. (https://doi.org/10.1038/nm.2173)

Kim SY, Sharma AJ \& Callaghan WM 2012 Gestational diabetes and childhood obesity: what is the link? Current Opinion in Obstetrics and Gynecology 24 376-381. (https://doi.org/10.1097/ GCO.0b013e328359fof4)

Kirwan JP, Hauguel-De Mouzon S, Lepercq J, Challier JC, HustonPresley L, Friedman JE, Kalhan SC \& Catalano PM 2002 TNF- $\alpha$ is a predictor of insulin resistance in human pregnancy. Diabetes $\mathbf{5 1}$ 2207-2213. (https://doi.org/10.2337/diabetes.51.7.2207)

Kirwan JP, Varastehpour A, Jing M, Presley L, Shao J, Friedman JE \& Catalano PM 2004 Reversal of insulin resistance postpartum is linked to enhanced skeletal muscle insulin signaling. Journal of Clinical Endocrinology and Metabolism 89 4678-4684. (https://doi.org/10.1210/ jc.2004-0749)

Kolb H \& Mandrup-Poulsen T 2005 An immune origin of type 2 diabetes? Diabetologia 48 1038-1050. (https://doi.org/10.1007/s00125-005-1764-9)

Lappas M, Permezel M \& Rice GE 2005 Leptin and adiponectin stimulate the release of proinflammatory cytokines and prostaglandins from human placenta and maternal adipose tissue via nuclear factorkappaB, peroxisomal proliferator-activated receptor-gamma and extracellularly regulated kinase 1/2. Endocrinology 146 3334-3342. (https://doi.org/10.1210/en.2005-0406) 
Li W, Zhang S, Liu H, Wang L, Zhang C, Leng J, Yu Z, Yang X, Tian H \& Hu G 2014 Different associations of diabetes with $\beta$-cell dysfunction and insulin resistance among obese and nonobese Chinese women with prior gestational diabetes mellitus. Diabetes Care 37 2533-2539. (https://doi.org/10.2337/dc14-0573)

Matsuda M, Mori T, Park MK, Yanaihara N \& Kawashima S 1994 Enhanced cell proliferation by hyperprolactinemia in both exocrine and endocrine pancreas in mice. European Journal of Endocrinology 130 187-194. (https://doi.org/10.1530/eje.0.1300187)

Meggyes M, Miko E, Szigeti B, Farkas N \& Szereday L 2019 The importance of the PD-1/PD-L1 pathway at the maternal-fetal interface. BMC Pregnancy and Childbirth 19 74. (https://doi.org/10.1186/s12884-019-2218-6)

Melchior H, Kurch-Bek D \& Mund M 2017 The prevalence of gestational diabetes. Deutsches Arzteblatt International 114 412-418. (https://doi. org/10.3238/arztebl.2017.0412)

Mirghani Dirar A \& Doupis J 2017 Gestational diabetes from A to Z. World Journal of Diabetes 8 489-511. (https://doi.org/10.4239/wjd.v8.i12.489)

Mitanchez D, Yzydorczyk C \& Simeoni U 2015 What neonatal complications should the pediatrician be aware of in case of maternal gestational diabetes? World Journal of Diabetes 6 734-743. (https://doi org/10.4239/wjd.v6.i5.734)

Molęda P, Homa K, Safranow K, Celewicz Z, Fronczyk A \& Majkowska L 2013 Women with normal glucose tolerance and a history of gestational diabetes show significant impairment of $\beta$-cell function at normal insulin sensitivity. Diabetes and Metabolism 39 155-162. (https://doi.org/10.1016/j.diabet.2012.11.003)

Mor G, Cardenas I, Abrahams V \& Guller S 2011 Inflammation and pregnancy: the role of the immune system at the implantation site. Annals of the New York Academy of Sciences 1221 80-87. (https://doi. org/10.1111/j.1749-6632.2010.05938.x)

Newbern D \& Freemark M 2011 Placental hormones and the control of maternal metabolism and fetal growth. Current Opinion in Endocrinology, Diabetes, and Obesity 18 409-416. (https://doi. org/10.1097/MED.0b013e32834c800d)

Nguyen CL, Pham NM, Binns CW, Van Duong D \& Lee AH 2018 Prevalence of gestational diabetes mellitus in eastern and southeastern Asia: a systematic review and meta-analysis. Journal of Diabetes Research 2018 1-10. (https://doi.org/10.1155/2018/6536974)

Pantham P, Aye ILMH \& Powell TL 2015 Inflammation in maternal obesity and gestational diabetes mellitus. Placenta 36 709-715. (https://doi.org/10.1016/j.placenta.2015.04.006)

Parsons JA, Brelje TC \& Sorenson RL 1992 Adaptation of islets of Langerhans to pregnancy: increased islet cell proliferation and insulin secretion correlates with the onset of placental lactogen secretion. Endocrinology 130 1459-1466. (https://doi.org/10.1210/endo.130.3.1537300)

Petra IL, Martín-Montalvo A, Cobo Vuilleumier N \& Gauthier BR 2019 Molecular modelling of islet $\beta$-cell adaptation to inflammation in pregnancy and gestational diabetes mellitus. International Journal of Molecular Sciences 20 6171. (https://doi.org/10.3390/ijms20246171)

Quesada-Candela C, Tudurí E, Marroquí L, Alonso-Magdalena P, Quesada I \& Nadal Á 2019 Morphological and functional adaptations of pancreatic alpha-cells during late pregnancy in the mouse. Metabolism: Clinical and Experimental 0 153963. (https://doi. org/10.1016/j.metabol.2019.153963)

Retnakaran R, Qi Y, Sermer M, Connelly PW, Hanley AJG \& Zinman B 2008 Glucose intolerance in pregnancy and future risk of pre-diabetes or diabetes. Diabetes Care 31 2026-2031. (https://doi.org/10.2337/ dc08-0972)

Rieck S \& Kaestner KH 2010 Expansion of $\beta$-cell mass in response to pregnancy. Trends in Endocrinology and Metabolism 21 151-158. (https://doi.org/10.1016/j.tem.2009.11.001)
Saisho Y, Miyakoshi K, Ikenoue S, Kasuga Y, Matsumoto T, Minegishi K, Yoshimura Y \& Itoh H 2013 Marked decline in beta cell function during pregnancy leads to the development of glucose intolerance in Japanese women. Endocrine Journal 60 533-539. (https://doi. org/10.1507/endocrj.EJ12-0356)

Scaglia L, Smith FE \& Bonner-Weir S 1995 Apoptosis contributes to the involution of beta cell mass in the post partum rat pancreas. Endocrinology 136 5461-5468. (https://doi.org/10.1210/ endo.136.12.7588296)

Sorenson RL \& Brelje TC 1997 Adaptation of islets of Langerhans to pregnancy: beta-cell growth, enhanced insulin secretion and the role of lactogenic hormones. Hormone and Metabolic Research 29 301-307. (https://doi.org/10.1055/s-2007-979040)

Sorenson RL, Brelje TC \& Roth C 1993 Effects of steroid and lactogenic hormones on islets of Langerhans: a new hypothesis for the role of pregnancy steroids in the adaptation of islets to pregnancy. Endocrinology 133 2227-2234. (https://doi.org/10.1210/ endo.133.5.8404674)

Szlapinski SK, King RT, Retta G, Yeo E, Strutt BJ \& Hill DJ 2019 A mouse model of gestational glucose intolerance through exposure to a low protein diet during fetal and neonatal development. Journal of Physiology 597 4237-4250. (https://doi.org/10.1113/JP277884)

Tang C, Han P, Oprescu AI, Lee SC, Gyulkhandanyan AV, Chan GNY, Wheeler MB \& Giacca A 2007 Evidence for a role of superoxide generation in glucose-induced beta-cell dysfunction in vivo. Diabetes 56 2722-2731. (https://doi.org/10.2337/db07-0279)

Van Assche FA, Aerts L \& De Prins F 1978 A morphological study of the endocrine pancreas in human pregnancy. British Journal of Obstetrics and Gynaecology 85 818-820. (https://doi. org/10.1111/j.1471-0528.1978.tb15835.x)

Veras E, Kurman RJ, Wang TL \& Shih IM 2017 PD-L1 expression in human placentas and gestational trophoblastic diseases. International Journal of Gynecological Pathology 36 146-153. (https://doi. org/10.1097/PGP.0000000000000305)

Wang CJ, Chou FC, Chu CH, Wu JC, Lin SH, Chang DM \& Sytwu HK 2008 Protective role of programmed death 1 ligand 1 (PD-L1) in nonobese diabetic mice: the paradox in transgenic models. Diabetes 57 1861-1869. (https://doi.org/10.2337/db07-1260)

Wedekind L \& Belkacemi L 2016 Altered cytokine network in gestational diabetes mellitus affects maternal insulin and placental-fetal development. Journal of Diabetes and its Complications 30 1393-1400. (https://doi.org/10.1016/j.jdiacomp.2016.05.011)

Westacott MJ, Farnsworth NL, St. Clair JR, Poffenberger G, Heintz A, Ludin NW, Hart NJ, Powers AC \& Benninger RKP 2017 Age-dependent decline in the coordinated $\left[\mathrm{Ca}^{2+}\right]$ and insulin secretory dynamics in human pancreatic islets. Diabetes 66 2436-2445. (https://doi. org/10.2337/db17-0137)

Xiang AH, Kawakubo M, Trigo E, Kjos SL \& Buchanan TA 2010 Declining beta-cell compensation for insulin resistance in hispanic women with recent gestational diabetes mellitus: association with changes in weight, adiponectin, and C-reactive protein. Diabetes Care 33 396-401. (https://doi.org/10.2337/dc09-1493)

Yang Y, Liu L, Liu B, Li Q, Wang Z, Fan S, Wang H \& Wang L 2018 Functional defects of regulatory $\mathrm{T}$ cell through interleukin 10 mediated mechanism in the induction of gestational diabetes mellitus. DNA and Cell Biology 37 278-285. (https://doi.org/10.1089/dna.2017.4005)

Ye X, Ju S, Duan H, Yao Y, Wu J, Zhong S, Chen L, Cao S, Xu Y, Zheng X, et al. 2017 Immune checkpoint molecule PD-1 acts as a novel biomarker for the pathological process of gestational diabetes mellitus. Biomarkers in Medicine 11 741-749. (https://doi.org/10.2217/ bmm-2017-0078)

Received in final form 20 February 2020

Accepted 13 March 2020

Accepted Manuscript published online 13 March 2020 https://joe.bioscientifica.com https://doi.org/10.1530/JOE-20-0012 (c) 2020 Society for Endocrinology Published by Bioscientifica Ltd. Printed in Great Britain 